\title{
Effects of Acute Transcutaneous Vagal Stimulation on the EEG Power Maps, EEG Sources Distribution and Steadiness of Quiet and Sensory-conflicted Stance
}

\section{Bozhidar Dimitrov ${ }^{a}$ and Plamen Gatev ${ }^{b}$}

$a$

\author{
Inst. of Population and Human Studies, BAS, Acad. G. Bonchev Str., BI.6, Sofia 1113, Bulgaria
}

${ }^{b}$ Institute of Neurobiology, BAS, Acad. G. Bonchev Str., BI. 23, Sofia 1113, Bulgaria

ABSTRACT: Transcutaneous vagal stimulation (t-VNS) is a prospective method as a cheap and noninvasive alternative of surgical cervical vagal stimulation widely used for treatment of refractory epilepsy, depression etc. Data on its application in healthy people are scarce. A lot of problems concerning the acting mechanisms and cortical representation are unsolved. We did acute t-VNS and studied the standing balance steadiness, EEG absolute power maps and sources distribution. We found improvement of the steadiness of standing with eyes open (EO), but no changes in the eyes closed (EC) series.

In EO Delta and Theta power in the right frontal area increased and Beta and Gamma bands of EEG absolute power in the left and right parieto-occipital areas also increased, which sustained for 20 min after $t-V N S$. The distribution of maxima of EEG sources revealed by LORETA showed shifts to the right and occipital direction after $t-V N S$, while 20 min later the maxima were symmetrically located, but the occipital shift was preserved. Pre-treatment sources during EO were in multisensory fields: the inferior parietal lobule, superior temporal gyrus and precuneus, after t-VNS they were in the inferior parietal lobule, middle temporal gyrus, right posterior cingulate, precuneus, cuneus and right lingual gyrus. After 20 min the maxima of EEG sources were in the inferior parietal lobule, superior and middle temporal gyrus and left insula.

In EC following t-VNS Delta power first decreased in the right frontal and occipital areas and in the central parietal area, 20 min later it was greater than in pre-treatment series in these areas. The results showed an increase of EEG absolute power in the Beta and Gamma ranges mainly in the occipital area and less expressed in the postero-parietal one. The increase was more expressed for Gamma than Beta band similarly to the EO series. However, this increase in Beta and Gamma EEG power in EC was mainly located in the left side of the cortex. After t-VNS we observed shifts of electrical sources locations to the occipital and top parts of the brain. Unlike, during the EO series we observed shift to the lower frequencies in the absolute power cortical maps. After 20 min a tendency to return to the pre-treatment coordinates occurred, the power, increased immediately after t-VNS, dropped much lower than its initial value. The locations of maxima of electrical sources during EC before t-VNS were in the middle and inferior frontal gyrus, right inferior parietal lobule, cingulate and precuneus. Immediately after t- 
VNS these locations were in the superior temporal gyrus, middle temporal gyrus, precuneus, cuneus and left inferior parietal lobule. After $20 \mathrm{~min}$ the locations of the maxima of electrical sources were in the anterior cingulate cortex, medial frontal gyrus, precuneus, cuneus and lingual gyrus.

Our results show that cortical processing of the t-VNS information and its effect on standing balance are quite different during standing with EO compared to standing with EC and that suggests differences due to the pairing of t-VNS with different sensory environment. This hypothesis can explain much of controversies connected with vagal stimulation in animals, healthy people and patients.

KEYWORDS: Standing balance, EEG spectra, LORETA, sensory conflict, t-VNS

\section{Introduction}

There is a fast growing interest in the transcutaneous vagal nerve stimulation (t-VNS), first proposed by Ventureira, ${ }^{1}$ which is a promising treatment of refractory epilepsy, pain, depression and other neuropsychiatric diseases ${ }^{2-5}$ and special stimulating devices are constructed for its application. ${ }^{6}$ This method represents a noninvasive, safer and cheaper alternative of the cervical vagal nerve stimulation, studies that started in 1938 with cat experiments of Bailey and Bremmer $^{7}$ and established the vagal nerve as an important route in the central nervous system, which can be used for brain neuromodulation by the nonspecific stimulation information carried by vagal afferents. ${ }^{8-9}$ Despite the long time and number of studies, the mechanisms of therapeutic action of both types of vagal nerve stimulation and its impact on cortical excitability are still unclear. The t-VNS electrical stimulation evokes brainstem potentials that can be recorded from scalp and used for diagnostic purposes. ${ }^{9-10}$ However, there is a strong debate going whether these potentials are from neurogenic ${ }^{11}$ or myogenic origin. ${ }^{12-13}$ Our aim was to test the effect of t-VNS on the quiet and sensory conflicted stance and the probable changes in the EEG spectral power maps and locations of the EEG electrical dipole sources by solving the inverse problem via LORETA method m-16 $^{14-}$ one fast, cheap and accurate tool to localize the sources of a given scalp record.

\section{Methods}

The study was approved by the local bioethics committee. The experimental task was standing on a pedobarographic platform Tekscan Evolution (Tekscan Inc., South Boston, MA, USA) shown on Fig. 1A, provided with Research Software and Sway Analysis Module (SAM) Matscan (Tekscan Inc., South Boston, MA, USA). Subjects stood as steady as possible with feet side-to-side and arms freely hanging alongside the body.

The electroencephalogram (EEG) was registered simultaneously with COP recording by 19 scalp electrodes attached on the scalp in accordance to the 10-20 system of the IFCN (Fig. 1D) on a 24 channel MITSAR 202 EEG machine (Mitsar Medical, Petersburg, Russia, Fig. 1C), with bandwidth 0.5-70 $\mathrm{Hz}$ and sampling rate of $500 \mathrm{~Hz}$. Following off-line artifacts' removal with the WINEEG software, current density sources were estimated with LORETA. We mapped the locations of maxima of current density 

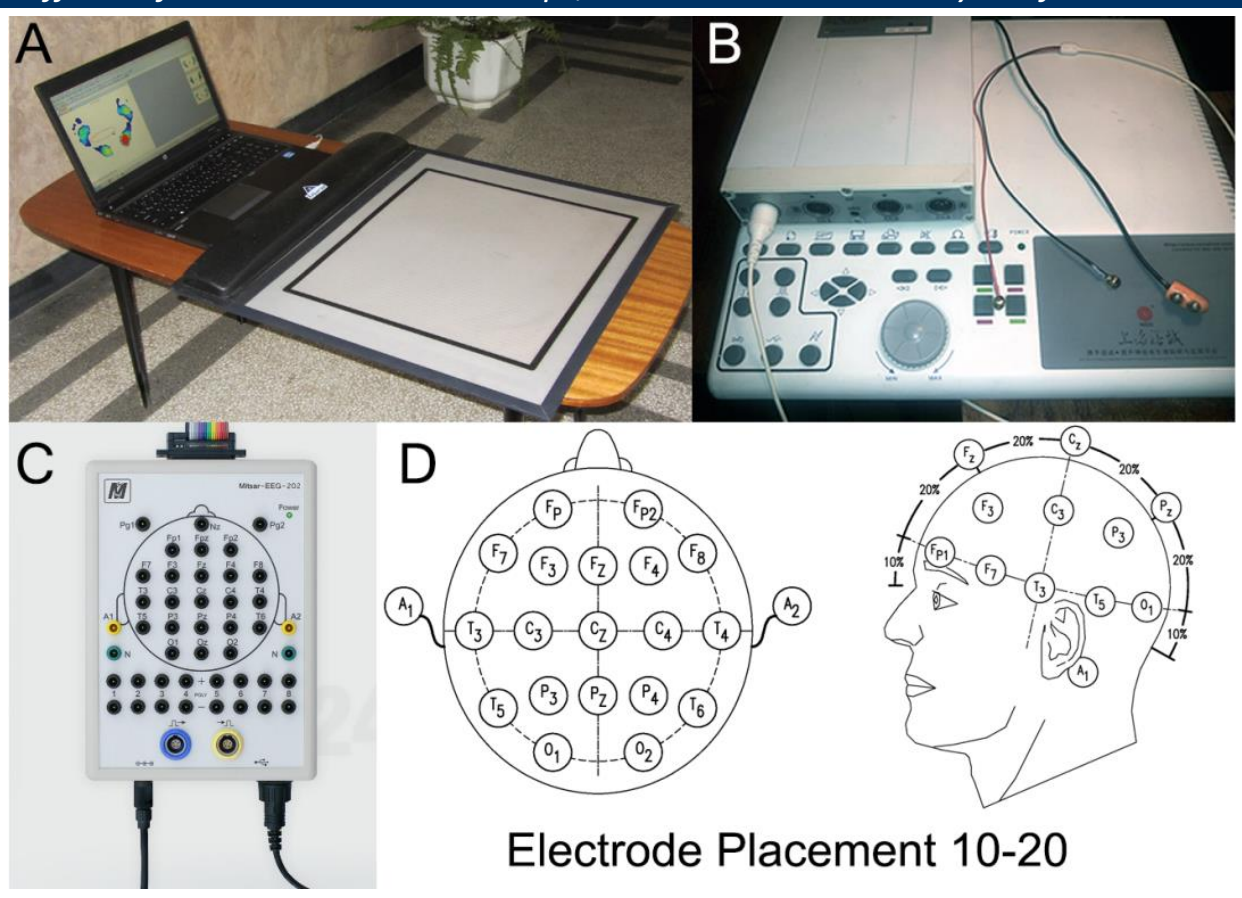

Electrode Placement 10-20

Figure 1: Experimental set. A: The laptop and pedobarographic platform. B: The 4-channel EMG-EP, stimulation and recording electrodes. C: The 32-channel EEG machine. D: The 10-20 EEG electrode montage.

of EEG sources before, immediately following and 20 minutes after the t-VNS during both eyes open (EO) and eyes closed (EC) series. The auricular rightside t-VNS was done by NTS-2000-B34: a 4-channel, portable EMG machine with EP function (NCC Medical Shanghai, China, Fig. 1B). First, we established the right place for electrical vagal stimulation with 8-10 $\mathrm{mA}$, rectangular pulses of $0.1 \mathrm{~ms}$ width, $0.5 \mathrm{~Hz}$ frequency; a bipolar EEG electrode - F4-P4 for feedback was used. Second, we did three $30 \mathrm{sec}$ records with the platform and EEG was recorded synchronously in two series - EO and EC with sufficient rest between them. After the rest we performed vagal electrical stimulation with electrodes on cymba conchae in four trains of 300 pulses with $4.1 \mathrm{~Hz}$ frequency, separated with $30 \mathrm{sec}$ rest between them. Immediately after stimulation and $20 \mathrm{~min}$ later, we did records as the pre-treatment ones. Cardiovascular parameters like blood pressure and pulse rate were monitored.

The results from the pedobarographic measurements (Fig.2) showed that the sway path of the center-ofpressure (COP) significantly diminished $20 \mathrm{~min}$ after $\mathrm{t}$-VNS stimulation during standing with EO. In the experimental series EC (Fig.2) we did not observe significant changes in the standing balance after $\mathrm{t}$-VNS.

\section{Results and discussion}

\subsection{Standing Balance}

The results from the pedobarographic measurements (Fig.2) showed that the 
sway path of the center-of-pressure (COP) significantly diminished 20 min after t-VNS stimulation during standing with EO. In the experimental series EC (Fig.2) we did not observe significant changes in the standing balance after $\mathrm{t}$ VNS.

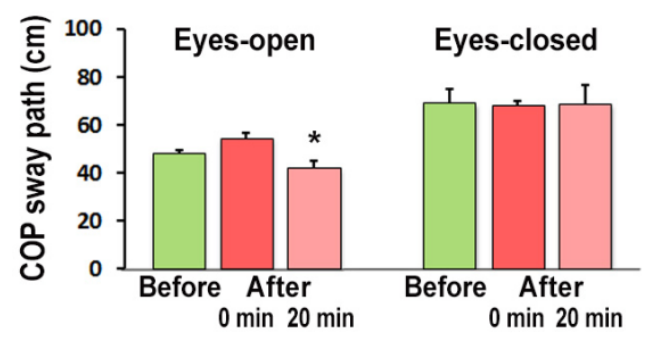

Figure 2: Means +/- SEM of the overall sway path of the COP during standing before, immediately after and $\mathbf{2 0}$ min later in two sensory conditions-EO and EC, $p<0.05$, statistically significant difference compared to the pre-treatment level.

\subsection{EEG measures during EO series}

The cortical maps of absolute power of EEG during the EO series for the slow Delta and Theta frequencies after the stimulation showed an increased power in the right frontal areas more expressed for the delta range, which might be due to some entrainment (Fig. 3, left panel). Alpha1 and Alpha2 frequencies after stimulation had a slight increase of power in the right parietal area. The greatest post-stimulation power changes we observed in the higher Beta and Gamma frequencies, representing an increase of the EEG absolute power in the left and right parieto-occipital areas immediately after t-VNS. In addition we observed also a post-stimulation dynamics in these ranges since $20 \mathrm{~min}$ after $\mathrm{t}$-VNS this increase of Beta and
Gamma EEG power remained localized in the right occipital cortical area (Fig. 3, left panel). Taking into account that this area is primarily connected with visual processing, this may explain the significant improvement of standing balance after $t-V N S$ during the EO series.

\subsection{EEG measures during EC series}

We have found significant changes in the absolute power cortical maps after t-VNS during the EC series, too. Delta power immediately after t-VNS decreased in the right frontal and occipital areas as well as in the central parietal areas, 20 min later it increased and became greater than in pre-treatment series in these areas (Fig.3, right panel). At this time a slight increase of Theta power was observed. There were no changes in the power in the Alpha1 and Alpha2 ranges. The results showed an increase of EEG absolute power in the Beta and Gamma ranges mainly in the occipital and less expressed in the postero-parietal area. The increase was more expressed for the Gamma than Beta band, similar to the EO series. However, unlike during EO, this increase in Beta and Gamma EEG power during the EC series was mainly located in the left side of the cortex and this may explain the difference between the series concerning the effect of $t-V N S$ on standing balance.

In general our findings confirm that changes in the cortex waves due to vagal stimulation are in the slow Delta and Theta waves ${ }^{18}$ (significant differences in Theta and Delta bands between healthy controls and patients with chronic pancreatitis following painful stimulation in the gut have been found) and in the high frequency Beta and Gamma bands. ${ }^{19-20}$ 
The data from LORETA during the EO series (Fig.4, left panel) showed shifts of EEG sources to the right and to the occipital direction immediately after t-VNS. Twenty minutes later the maxima were symmetrically situated but the occipital shift was preserved.

The locations of maxima of the EEG sources before t-VNS were in the inferior parietal lobule, superior temporal gyrus and precuneus. Immediately after stimulation they were in the inferior parietal lobule, middle temporal gyrus, right posterior cingulate, precuneus, cuneus and right lingual gyrus.

Twenty minutes after t-VNS the locations of maxima of EEG sources were in the superior temporal gyrus, middle temporal gyrus, inferior parietal lobule and left insula (Fig.4, left panel).

It is interesting to compare our results with the findings of Kosel et al. ${ }^{17}$ that regional blood flow (rCBF) after chronic cervical vagal stimulation for adjunctive treatment of depressed patients was increased in the left dorsolateral/ventrolateral prefrontal cortex (Brodmann areas 46 and 47) and decreased in the right posterior cingulate area, the lingual gyrus and the left insula. The similarities concerning locations and differences might be due to the chronic vs. acute stimulation.

During the EC series (Fig. 4, right panel) immediately after the t-VNS we observed an ensuing t-VNS shift to the left unlike during the EO series, which was similar to the changes in the absolute power cortical maps. Another shift was towards the top. Twenty minutes after $\mathrm{t}$-VNS there was a tendency to return to the pre-treatment coordinates and the increased power immediately after stimulation dropped even much lower than its initial value.

The locations of maxima of electrical sources during series EC before t-VNS were in the middle and inferior frontal gyrus, right inferior parietal lobule, cingulate and precuneus.

Immediately after stimulation these locations were in the superior temporal gyrus, middle temporal gyrus, precuneus, cuneus and left inferior parietal lobule. Twenty minutes after stimulation the locations of the maxima of electrical sources were observed in the anterior cingulate cortex, medial frontal gyrus, precuneus, cuneus and lingual gyrus.

Our findings suggest in principal that the t-VNS may improve steadiness of standing balance with EO when all senses are available.

However, further investigations are necessary to establish the duration of the effect and how different stimulation parameters would affect it.

Our results support the hypothesis that the t-VNS influences cortical structures involved in the processing of afferent vagal flow ${ }^{21}$, especially specific ones like insula and lingual gyrus. It is interesting to accentuate the differences found between EO and EC series concerning the standing balance, the cortical absolute power maps and the locations of the maxima of electrical sources, which suggest that the effect of t-VNS may be different due to sensory conflict when visual information is absent. The results are generally in line with findings of Drew et al. ${ }^{20}$ Following a painful esophageal stimulation they have found reliable principal components in the thalamus, insula, cingulate gyrus, and in several regions in the sen- 
sory cortex and dynamic cross-coherence links. In the cingulate gyrus, the posterior areas were always activated first, followed by the middle and anterior regions in the Beta $(14-25 \mathrm{~Hz})$ and Gamma (25-50 Hz) frequency bands between networks of neurones. Our findings confirm also that t-VNS is a safe, cheap and effective way to stimulate vagal nerve and provides innovative data about the possible mechanisms of action in healthy people during quiet and sensory-conflicted stance. They revealed a potential for application of this technique for cognitive improvement, which is rather scarcely researched. ${ }^{22-23}$ Despite of the long history of vagal stimulation studies in animals and people, the mechanisms of its therapeutic effect are still unclear. ${ }^{24}$ The vagal stimulation used for neuromodulation evokes both synaptic and non-synaptic diffusion neurotransmission. ${ }^{25-26}$ Furthermore, the neuromodulation effects depend on the stimulation conditions like site of stimulation, frequency, amplitude, width of stimulus, whether large myelinated or small non-myelinated nerve fibers are involved, etc. among many other parameters.

It is generally accepted that afferent vagal stimulation including t-VNS influences cortical plasticity. However, a debate has started whether synaptic neurotransmission reaching the cortex in the form of evoked cortical potentials were myogenic artefacts.

Animal experiments showed that at least the first responses of shortest latency did not disappear after general anesthesia. $^{27}$

Recently a new hypothesis has been raised. It supposes that targeted cortical plasticity can be obtained by pairing vagal stimulation (producing task non-specific modality afferent information only) with a second stimulation (producing task specific modality afferent information) ${ }^{28-32}$ (See a recent review by Hays et al., $2013^{33}$ ). In order to enhance cortical plasticity it is important that the vagal stimulation and the sensory or motor event are paired in time precisely.

The most interesting and important of our findings is that cortical processing of the t-VNS afferent information and its effect on standing balance were quite different during standing with EO compared with standing with EC. This suggests differences due to the pairing of $t$ VNS with different sensory environment. Such hypothesis can explain much of controversies connected with vagal stimulation in animals, healthy people and patients. For example, the disappearance of vagal cortical potentials ${ }^{9-11}$ in healthy and conscious people after neuromuscular block due to anesthesia ${ }^{12-13,27}$ might not be easily explained by the supposition that they are from myogenic origin, but with the fact of the changed sensory environment in this case.

It is noteworthy that the main function of the vagal nerve, called also the Great Wanderer, is to keep the homeostasis $^{33}$ and this might be done in many ways depending on the context found during its wandering: fighting with enemies to break the pathological patterns like in patients ${ }^{6}$ or supporting the friends like an increase of cognitive abilities in healthy people $22-23$ or doing nothing, thus not disturbing the fragile balance of homeostasis. 


\section{Acknowledgements}

The authors are thankful for the financial support provided by Grant TK 02/60 with the National Science Fund, Ministry of Education, Youth and Science, Republic of Bulgaria.

\section{Eyes-open}
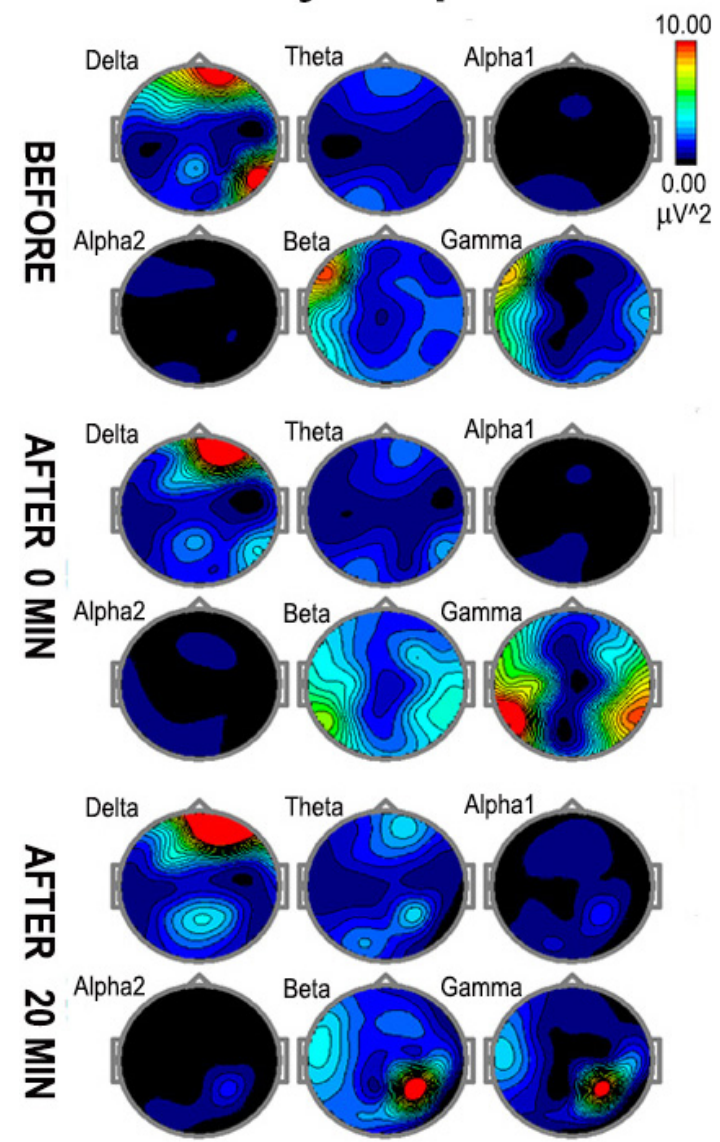

Eyes-closed
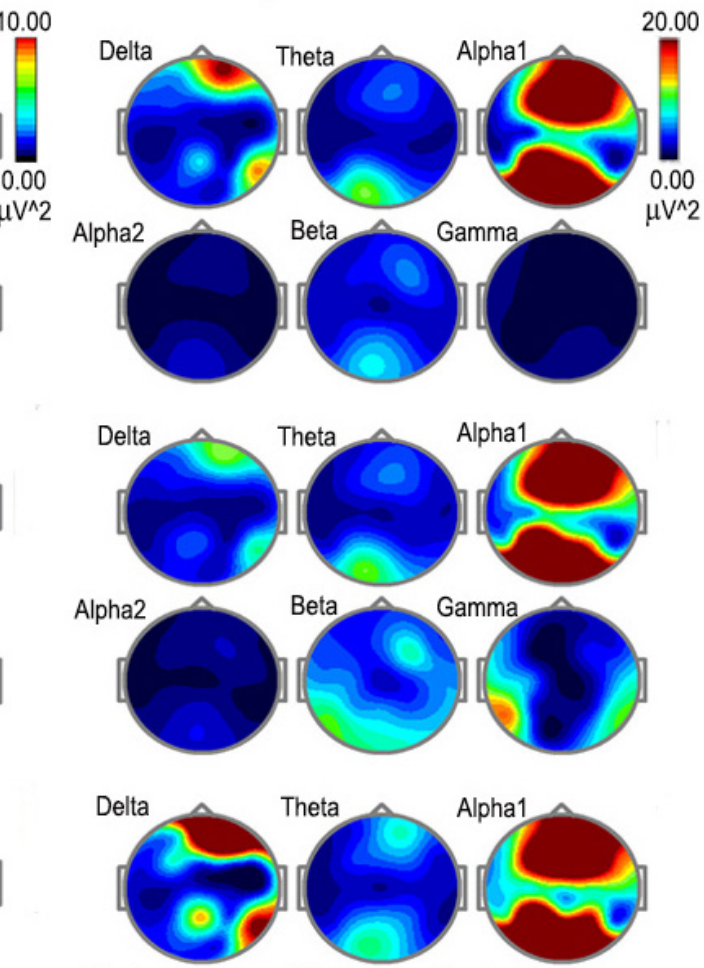

Alpha2

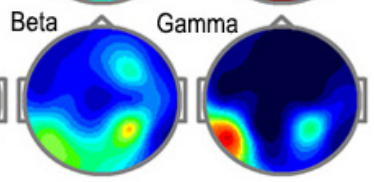

Figure 3: Cortical maps of absolute EEG power in the bands: Delta $(0.5-4 \mathrm{~Hz})$, Theta $(4-8 \mathrm{~Hz})$, Alpha1 (8-10 Hz), Alpha2 (10-12 Hz), Beta (12-30 Hz), and Gamma (30-60 Hz). Left panel Standing with eyes-open, Right panel - Standing with eyes-closed; Top rows - before t-VNS, middle rows - Immediately after and bottom rows - $20 \mathrm{~min}$ after $\mathrm{t}-\mathrm{VNS}$. Please note different color bars and the twofold decreased sensitivity during EC. 
Eyes-open

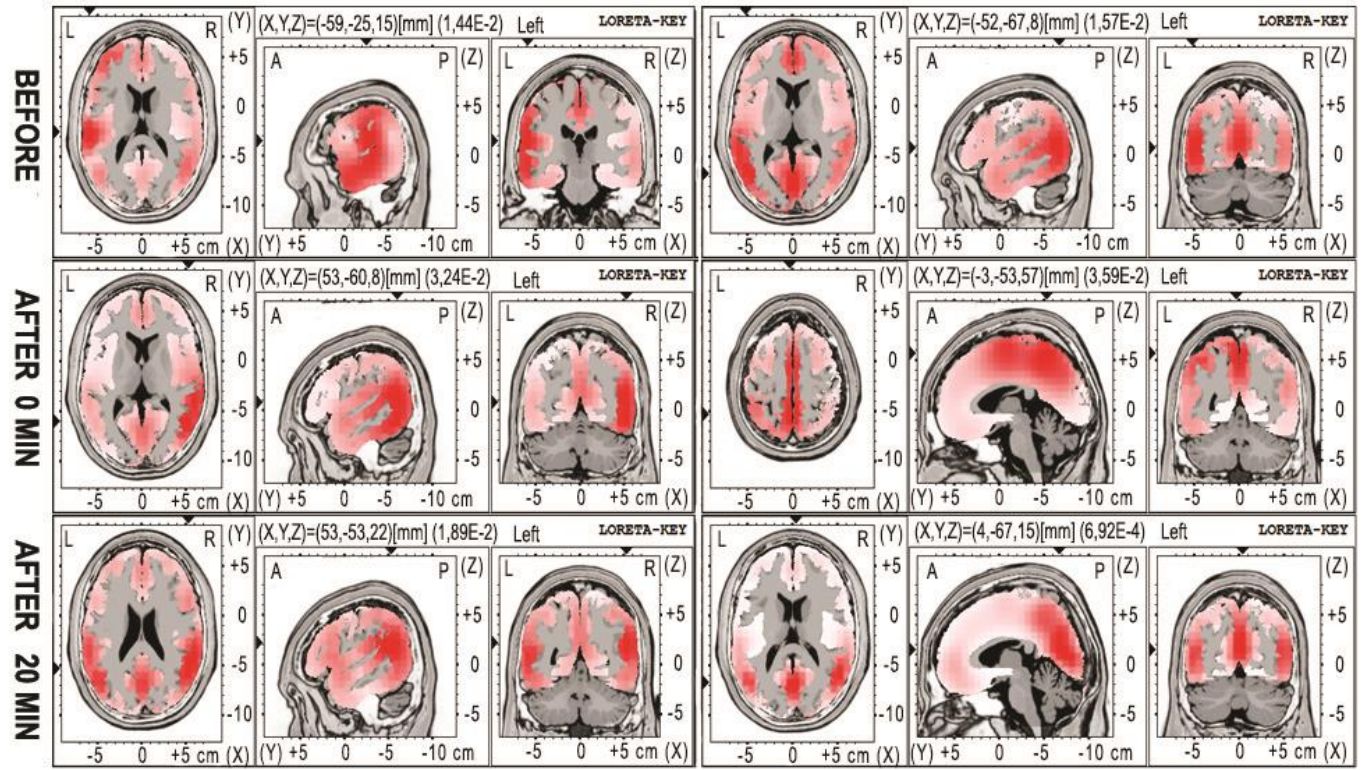

Figure 4: Data from LORETA: Left panel - Standing with eyes-open; Right panel - Standing with eyes-closed; Top rows - before t-VNS, middle rows - Immediately after and bottom rows $\mathbf{- 2 0}$ min after t-VNS.

\section{References}

1. Ventureyra EC. Transcutaneous vagus nerve stimulation for partial onset seizure therapy. A new concept. Childs Nerv Syst. 2000 Feb;16(2):101-2. doi: $10.1007 / \mathrm{s} 003810050021$.

2. Stefan $H$, Kreiselmeyer G, Kerling F, et al. Transcutaneous vagus nerve stimulation (t-VNS) in pharmacoresistant epilepsies: a proof of concept trial. Epilepsia. 2012 Jul;53(7): e115-8. doi: 10.1111/ j.1528-1167.2012.03492.x.

3. He $\mathbf{W}$, Jing $X$, Wang $X$, et al. Transcutaneous auricular vagus nerve stimulation as a complementary therapy for pediatric epilepsy: a pilot trial. Epilepsy Behav. 2013 Sep;28(3):343-6. doi: 10.1016/j.yebeh.2013.02.001.
4. Hein E, Nowak M, Kiess O, et al. Auricular transcutaneous electrical nerve stimulation in depressed patients: randomized controlled pilot study. J Neural Transm. 2013 May; 120(5):821-7. doi: 10.1007/s00702-012-0908-6.

5. Busch V, Zeman F, Heckel A, et al. The effect of transcutaneous vagus nerve stimulation on pain perception an experimental study. Brain Stimul. 2013 Mar;6(2):202-9. doi: 10.1016/j.brs. 2012.04.006.

6. Ben-Menachem E, Revesz D, Simon BJ, et al. Surgically implanted and noninvasive vagus nerve stimulation: a review of efficacy, safety and tolerability. Eur J Neurol. 2015 Jan 23. doi: 10.1111/ ene.12629. 
7. Bailey P, Bremmer FA. A sensory cortical representation of the vagus nerve with a note on the low pressure on the surface electrogram. Journal of Neurophysiology. 1938;1:405-412.

8. Schachter SC. Review of the mechanisms of action of antiepileptic drugs. CNS Drugs. 1995 Dec;4(6):469-77. doi: 10.2165/00023210-199504060-00009.

9. Fallgatter AJ, Polak T, Metzger F, et al. Brainstem Vagus nuclei evoked potentials - a new diagnostic method in neuropsychiatry? Nervenheilkunde. 2006;25: 669-73.

10. Polak T, Dresler T, Zeller JB, et al. Vagus somatosensory evoked potentials are delayed in Alzheimer's disease, but not in major depression. Eur Arch Psychiatry Clin Neurosci. 2014 Apr;264(3): 263-7. doi: 10.1007/s00406-013-0415-2.

11. Polak T, Metzger FG, Deckert J, et al. Central neural versus peripheral muscular origin of vagus somatosensoryevoked potentials. Brain Stimul. 2014 Jul-Aug; 7(4):624-5. doi: 10.1016/j.brs. 2014.04.003.

12. Leutzow B, Lange J, Gibb A, et al. Vagal sensory evoked potentials disappear under the neuromuscular block an experimental study. Brain Stimul. 2013 Sep;6(5):812-6. doi: 10.1016/j.brs. 2013.03.005.

13. Leutzow B, Nowak A, Usichenko TI. On the origin of scalp responses - a comment on the letter of Polak et al. Brain Stimul. 2014 Jul-Aug;7(4):625-6. doi: 10.1016/j.brs.2014.05.006.

14. Pascual-Marqui RD, Michel CM, Lehmann D. Low resolution electromagnetic tomography: a new method for localizing electrical activity in the brain. Int
J Psychophysiol. 1994 Oct;18(1):49-65. doi: 10.1016/0167-8760(84)90014-X.

15. Michel CM, Murray MM, Lantz G, et al. EEG source imaging. Clin Neurophysiol. Clin Neurophysiol. 2004 Oct;115(10): 2195-222. doi: 10.1016/j.clinph.2004.06. 001.

16. Pascual-Marqui RD. Review of methods for solving the EEG inverse problem. Int J Bioelectromagnetism. 1999;1(1):75-86.

17. Kosel M, Brockmann $H$, Frick $C$, et al. Chronic vagus nerve stimulation for treatment-resistant depression increases regional cerebral blood flow in the dorsolateral prefrontal cortex. Psychiatry Res. 2011 Mar 31;191(3):153-9. doi: 10.1016/j.pscychresns.2010.11.004.

18. Drewes AM, Gratkowski M, Sami $\mathrm{SA}$, et al. Is the pain in chronic pancreatitis of neuropathic origin? Support from EEG studies during experimental pain. World J Gastroenterol. 2008 Jul 7; 14 (25):4020-7. doi:10.3748/wjg.14.4020.

19. Hyvärinen P, Yrttiaho S, Lehtimäki J, et al. Transcutaneous vagus nerve stimulation modulates tinnitus-related Betaand Gamma-band activity. Ear Hear. 2014 Nov 26. [Epub ahead of print] doi: 10.1097/AUD.0000000000000123.

20. Drewes AM, Sami SAK, Dimcevski $G$, et al. Cerebral processing of painful oesophageal stimulation: a study based on independent component analysis of the EEG. Gut. 2006 May;55(5):619-29. doi: 10.1136/gut.2005.068460.

21. Sharma A, Lelic D, Brock C, et al. New technologies to investigate the brain-gut axis. World Journal of Gastroenterology: WJG 2009 Jan14;15(2):18291. doi: 10.3748/wjg.15.182. 
22. Clark KB, Naritoku DK, Smith DC, et al. Enhanced recognition memory following vagus nerve stimulation in human subjects. Nat Neurosci. 1999 Jan; 2(1):94-8. doi: 10.1038/4600.

23. Van Leusden JWR, Sellaro R, Colzato LS. Transcutaneous vagal nerve stimulation (tVNS): a new neuromodulation tool in healthy humans? Front Psychol. 2015 Feb 10. doi: 10.3389/fpsyg. 2015.00102.

24. Henry TR. Therapeutic mechanisms of vagus nerve stimulation. Neurology. 2002 Sep 24;59(6 Suppl 4):S3-14.

25. Craig AD. How do you feel? Interoception: the sense of the physiological condition of the body. Nat Rev Neurosci. 2002 Aug;3(8):655-66. doi: 10.1038/ nrn894.

26. Mayer EA. Gut feelings: the emerging biology of gut-brain communication. Nat Rev Neurosci. 2011 Jul 13; 12(8):453-66. doi: 10.1038/nrn3071.

27. Usami K, Kawai K, Sonoo M, et al. Scalp-recorded evoked potentials as a marker for afferent nerve impulse in clinical vagus nerve stimulation. Brain Stimul. 2013;6:615-23. doi: 10.1016/ j.brs.2012.09.007.

28. Engineer ND, Riley JR, Seale JD, et al. Reversing pathological neural activity using targeted plasticity. Nature. 2011
Feb 3;470(7332):101-4. doi: 10.1038/ nature09656.

29. Porter BA, Khodaparast N, Fayyaz T, et al. Repeatedly pairing vagus nerve stimulation with a movement reorganizes primary motor cortex. Cereb Cortex. 2012 Oct;22(10):2365-74. doi: 10.1093/ cercor/bhr316.

30. Shetake JA, Engineer ND, Vrana $W A$, et al. Pairing tone trains with vagus nerve stimulation induces temporal plasticity in auditory cortex. Exp Neurol. 2012 Jan;233(1):342-9. doi: 10.1016/ j.expneurol.2011.10.026.

31. Khodaparast N, Hays SA, Sloan AM, et al. Vagus nerve stimulation during rehabilitative training improves forelimb strength following ischemic stroke. Neurobiol Dis. 2013 Dec;60:80-8. doi: 10.1016/j.nbd.2013.08.002.

32. Engineer CT, Engineer ND, Riley JR, et al. Pairing speech sounds with vagus nerve stimulation drives stimulus-specific cortical plasticity. Brain Stimulation. In press. doi: 10.1016/j.brs.2015.01.408.

33. Hays SA, Rennaker RL, Kilgard MP. Targeting plasticity with vagus nerve stimulation to treat neurological disease. Prog Brain Res. 2013;207:275-99. doi: 10.1016/B978-0-444-63327-9.0001 0-2. 